\title{
BMJ Open Evaluation of gender differences in postoperative sore throat and hoarseness following the use of Ambu AuraGain laryngeal mask: the randomised controlled LadyLAMA trial study protocol
}

Katharina Epp (D) , ${ }^{1}$ Uli Przybylski, ${ }^{2}$ Carla Luz, ${ }^{2}$ Marc Kriege (D) , ${ }^{1}$ Eva Wittenmeier, ${ }^{1}$ Irene Schmidtmann, ${ }^{3}$ Nina Pirlich (i) ${ }^{1}$

To cite: Epp K, Przybylski U, Luz C, et al. Evaluation of gender differences in postoperative sore throat and hoarseness following the use of Ambu AuraGain laryngeal mask: the randomised controlled LadyLAMA trial study protocol. BMJ Open 2022;12:e056465. doi:10.1136/ bmjopen-2021-056465

- Prepublication history for this paper is available online To view these files, please visit the journal online (http://dx.doi. org/10.1136/bmjopen-2021056465).

Received 15 August 2021 Accepted 22 December 2021

\section{Check for updates}

(C) Author(s) (or their employer(s)) 2022. Re-use permitted under CC BY-NC. No commercial re-use. See rights and permissions. Published by BMJ.

${ }^{1}$ Anaesthesiology, University Medical Centre of the Johannes Gutenberg University Mainz, Mainz, Germany

${ }^{2}$ University Medical Centre of the Johannes Gutenberg University Mainz, Mainz, Germany ${ }^{3}$ Institute of Medical Biostatistics, Epidemiology and Informatics, University Medical Centre of the Johannes Gutenberg University Mainz, Mainz, Germany

Correspondence to Dr Katharina Epp; kathaepp@uni-mainz.de

\section{ABSTRACT}

Introduction Postoperative sore throat (POST) is a comparatively minor but very common side effect of general anaesthesia with a supraglottic airway device. The patient considers these side effects a mirror of the quality of anaesthesia. The aims of this study are to evaluate genderspecific differences in the incidence of POST and to assess whether the effects of known risk factors vary between genders.

Methods and analysis The LadyLAMA trial is a single-centre, patient-blinded, randomised controlled trial. Consecutive patients requiring ophthalmological surgery under general anaesthesia with a second generation Ambu AuraGain laryngeal mask are randomly allocated to either cuff pressure of $45 \mathrm{cmH} 20$ or cuff pressure of $60 \mathrm{cmH} 20$. We estimate the difference in POST between the genders at 20\% and we hypothesised that a reduction of cuff pressure would reduce POST by $10 \%$. A total of 800 patients will be recruited, with each subgroup including 200 patients to achieve $80 \%$ power for detecting a difference at the $5 \%$ significance level. Primary endpoints are gender differences in the incidence of POST within 24 hours postoperatively, as well as comparison of cuff pressure $45 \mathrm{cmH} 20$ to $60 \mathrm{cmH} 20$ with respect to POST. The main secondary objective is the effect of cuff pressure on POST stratified by gender. Further secondary endpoints are gender-specific differences in POST and hoarseness in postanaesthesia care unit (PACU) at 48 and 72 hours (or until freedom of discomfort). The parameter cuff pressure serves as key-secondary endpoint.

Ethics and dissemination The project is approved by the local ethics committee of the Medical Association of the Rhineland Palatine state (Nr. 2021-15835). The results of this study will be made available in the form of manuscripts for publication and presentations at national and international meetings.

Trial registration number NCT04915534.

\section{INTRODUCTION}

\section{Background and rationale}

General anaesthesia (GA) requires for the anaesthesiologist to secure the airway of the patient in order to ensure ventilation of the lungs, and therefore, oxygenation of the
Strengths and limitations of this study

- This is the first trial to evaluate the incidence of postoperative sore throat (POST) according to gender after using a second generation laryngeal mask.

- This trial aims to determine whether there is a genderspecific difference in the occurrence of POST and hoarseness under consideration of the cuff pressure.

- The study plan is to include 800 adult patients in a single-centre, patient-blinded and randomised controlled (superiority) study.

- Only one type of second-generation laryngeal mask is evaluated, therefore the results cannot be transferred to other kinds of laryngeal masks (eg, firstgeneration laryngeal masks, i-gel).

organs. Depending on the type of surgery and individual patient characteristics, either an endotracheal tube or a supraglottic airway device (SAD) can be used. Naturally, these airway devices suppress and irritate anatomical structures and can, therefore, lead to trouble swallowing, hoarseness and sore throat postoperatively. ${ }^{1}$ The incidence of postoperative sore throat (POST) and hoarseness following GA via laryngeal masks (LM) ranges between $12 \%$ and $49 \% .^{2-6}$ Following tracheal intubation, the incidence of POST is slightly higher in about $62 \%$ of cases. ${ }^{2}$

Since the development of the first prototype of the classic SAD by Brain, there have been numerous advancements of the original model by different manufacturers. ${ }^{7}$ Today, SADs are an essential component of routine GA. However, they are also a key element of the algorithms designed for the anticipated and unexpected difficult airway in both, national and international anaesthesiological societies. ${ }^{89}$ SADs are an effective alternative to endotracheal intubation because they do 
not require neuromuscular blockage or visualisation of the glottis. As previously described, they have a slightly lower incidence of POST than endotracheal intubation ${ }^{2}$ and in addition they reduce airway morbidity and have a positive effect on haemodynamics. ${ }^{10}$ Models of SADs differ according to the type of Cuff, the option of insertion of a gastric tube (second generation $\mathrm{SAD}$ ) and configuration of the ventilation tube.

The Ambu AuraGain (Ambu, Ballerup, Denmark) $\mathrm{LM}$ is an advanced version of SAD. The difference is a channel that allows the placement of a gastric tube, as well as an integrated anti-bite protection and the option to perform endotracheal intubation. The AuraGain provides improved safety against gastric regurgitation and aspiration. ${ }^{2}$ According to the manufacturer, it is anatomically designed and at the moment it is the first choice SAD used at University Medical Center Mainz.

The human larynx shows gender-specific differences. The laryngeal cartilage consists of two parts, which are connected in the anterior area. In males, they form an angle of about $90^{\circ}$, whereas in females the angle is about $120^{\circ}$. These differences are also visible from the outside, in the shape of the so-called male Adam's apple. In males, vocal cords are one quarter longer and thicker than in females and influence the sound of the voice for example. ${ }^{11}$ MR tomography scans show that the structures surrounding the larynx are anatomically significantly smaller in women than in men. ${ }^{12}$ However, the compliance of the larynx in men is higher than in women. ${ }^{13}$ Furthermore, a positive influence of female hormones on the genioglossus muscle, which originates at hyoid and epiglottis, is discussed. ${ }^{14}$ This muscle prevents the tongue from reverting to the pharynx during sleep and plays an important role in the genesis of sleep apnoea syndrome. ${ }^{14}$

The genesis of POST is multifactorial. Studies show a similar incidence of POST following GA with volatile anaesthetics in $14 \%-50 \%$, and in $27 \%-50 \%$ in total intravenous anaesthesia (TIVA), respectively. ${ }^{15} 16$ However, there are no superiority trials clearly stating an advantage of either anaesthetic agent regarding POST. Mencke et al were not able to show a benefit of Sevoflurane over TIVA in the occurrence of POST or laryngeal injury. ${ }^{16}{ }^{17}$ Insertion technique is also an important factor in the onset of POST. $^{2}$ There is a variety of different recommendations for inserting an SAD. Blocking the cuff previous to insertion is supposed to decrease the development of POST. ${ }^{18}$ Multiple attempts to place the $\mathrm{SAD}$, as well as using the index finger to help pass the hard palate have been associated with an increase of POST. ${ }^{219}$ Medication given in order to prevent postoperative nausea and vomiting, as well as postoperative pain may also be an influencing factor of POST. Several meta-analysis suggest, that applying intravenous dexamethasone may decrease the incidence of sore throat postoperatively. ${ }^{20}{ }^{21}$ In addition, we assume that the experience of the provider inserting the SAD may also influence POST. The S1 guideline for preclinical airway management suggests a number of 45 placements of an SAD in order to develop a routine in the application. ${ }^{9}$ We hypothesise that inexperienced providers cause increased rates of POST, because multiple insertion attempts are considered a risk factor. ${ }^{22}$

According to literature, the most important factor to influence the occurrence of POST is cuff pressure because the cuff may cause severe pressure damage to the point of necrosis and nerve lesions. ${ }^{23}$ The manufacturer's recommendation is a cuff pressure $<60 \mathrm{cmH} 2 \mathrm{O}$. Studies with first generation SAD show that a decrease in cuff pressure to $40-45 \mathrm{mmH} 2 \mathrm{O}$ significantly decreases the incidence of POST..$^{22} 24$ Nott et al were able to show that reducing the cuff pressure in female patients resulted in 2/3 decrease of POST ${ }^{22}$ However, this trial evaluated first generation $\mathrm{SAD}$ in 839 patients and the focus was on the cuff pressure, rather than the gender.

Despite POST being a comparatively minor adverse effect following GA, studies prove it is also a common side effect, which conveys the quality of the GA to the patient. ${ }^{3}$ Data collected from the internal data acquisition and accounting system (DAQ) of the University Medical Centre Mainz, show that the use of SAD increased in the past 15 years by about $200 \%$ because of the extended indication profile of SAD for patients with mild gastral reflux or special positioning for surgery. Despite gender-specific differences in airway anatomy, ${ }^{12}$ AuraGain, as well as any other SAD currently on the market, is only available in a unisex version.

To this date, there are no data comparing genderspecific differences in POST under consideration of cuff pressure after using second generation LM. The aim of this study is to compare incidence of POST and hoarseness between the genders under incorporation of the cuff pressure. Furthermore, we aim to identify risk factors that cause POST in both groups. According to existing studies, ${ }^{122}$ we hypothesise that women will suffer more from postoperative complications following GA than men, furthermore, we expect a lower cuff pressure to lower the incidence of POST.

\section{Study aims and objectives}

Primary objectives: First, we will be comparing the gender-specific incidence of POST between males and females within the first 24 hours after GA with a secondgeneration Ambu AuraGain LM for elective, ophthalmological surgery.

Second, the incidence of POST within the first 24 hours after use of Ambu AuraGain LM with cuff pressure of 45 $\mathrm{cmH} 2 \mathrm{O}$ and $60 \mathrm{cmH} 2 \mathrm{O}$ will be compared.

Primary endpoint in both cases is the incidence of POST.

Secondary objective: The effect of cuff pressure stratified by gender on POST within the first 24 hours postoperatively, as well as prevalence of POST and hoarseness in postanaesthesia care unit (PACU), at 48 and 72 hours until freedom of symptoms will be evaluated under consideration of influencing coefficients cuff pressure (45 $\mathrm{cmH} 2 \mathrm{O}$ and $60 \mathrm{cmH} 2 \mathrm{O}$ ) and gender. 


\section{METHODS AND ANALYSIS}

This manuscript was written in accordance with the Standard Protocol Items: Recommendations for Interventional Trials guidelines. ${ }^{26}$

\section{Study design and setting}

The LadyLAMA trial is a single centre, randomised controlled trial and performed in the operating room on patients undergoing ophthalmological surgery at University Medical Centre of Johannes-Gutenberg University in Mainz. All LMs are inserted by anaesthetists with different levels of clinical experience (residents, experts, number of LM inserted). After a specific introduction to the study protocol, all anaesthesiologists assigned to the section of ophthalmological surgery will participate in this trial. We have chosen to include only patients undergoing ophthalmological surgery because of a low postoperative pain profile, as well as short durations of surgery and anaesthesia.

\section{Eligibility criteria}

\section{Inclusion criteria}

Patients 18 years and older, requiring elective ophthalmological surgery in GA with a second-generation Ambu AuraGain LM, who have consented orally and in writing.

\section{Exclusion criteria}

- Anticipated difficult airway with indication for awake tracheal intubation.

- Indication for rapid sequence induction (RSI).

- Pregnant or breast feeding.

- Age $<18$ years.

- Obesity.

- Limited mouth opening.

- Pre-existing hoarseness.

- Out-patient surgery

- Unable to provide informed written consent or under guardianship.

\section{Patient population and allocation}

Patient recruitment is planed between August 2021 and December 2022. The history and physical examinations of all patients scheduled for surgery are screened preoperatively for exclusion criteria. Patient recruitment is conducted by one of the study physicians. After eligibility is confirmed and written informed consent is obtained, enrolled participants are randomised 24 hours before the intervention. A web-based service (QuickCalcs, GraphPad Software, La Jolla, California, USA) is used for allocating patients to either cuff pressure $45 \mathrm{cmH} 2 \mathrm{O}$ or cuff pressure $60 \mathrm{cmH} 2 \mathrm{O}$. The schedule of enrolment and intervention is shown in figure 1 , and the participant timeline is described in table 1 .

\section{Sequence generation}

The randomisation sequence of the cuff pressure (45 $\mathrm{cmH} 2 \mathrm{O}$ and $60 \mathrm{cmH} 2 \mathrm{O}$ ) is generated via block randomisation using the computer programme http://wwwgraphpadcom/quickcalcs/randMenu/. Allocation of the cuff
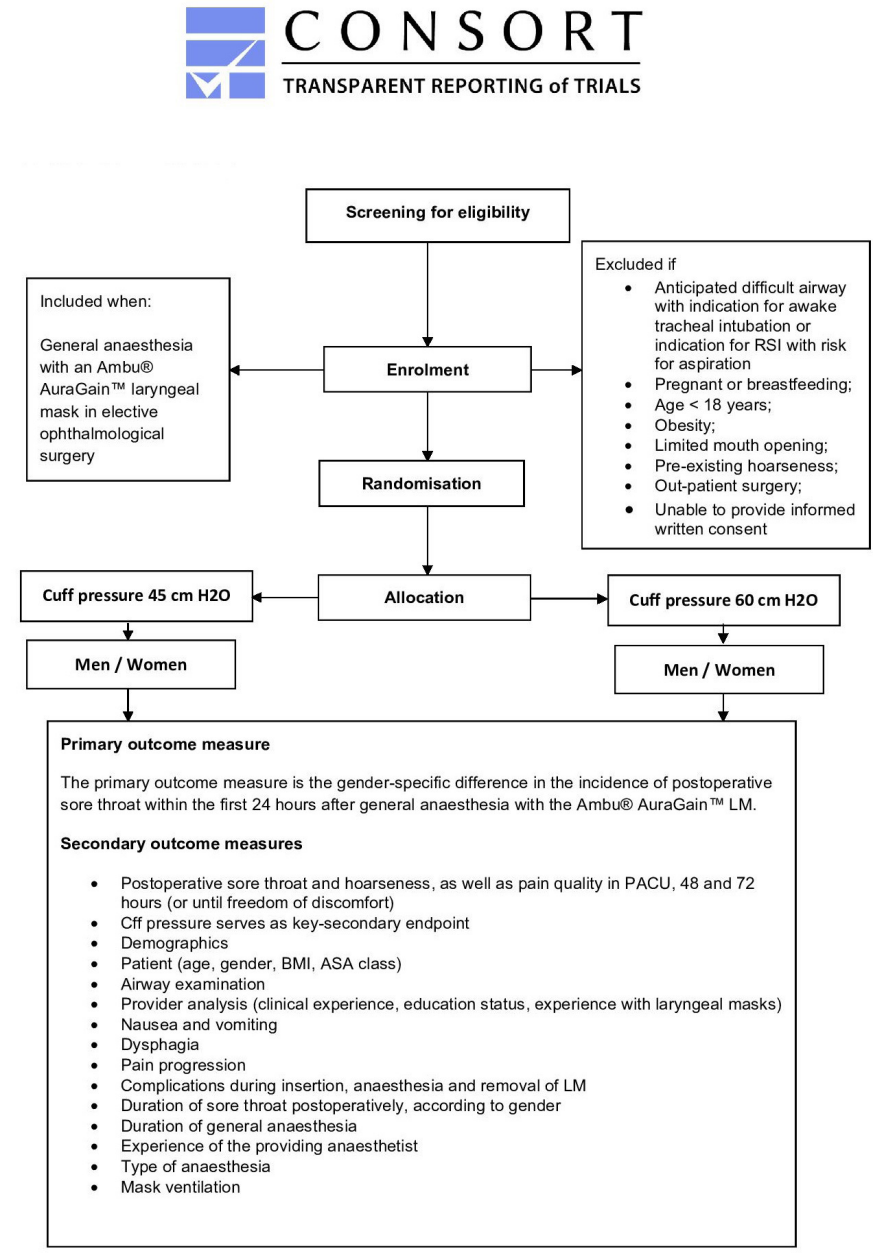

Figure 1 Study flow chart of recruitment and intervention schedule according to CONSORT guidelines. BMI, body mass index; CONSORT, Consolidated Standards of Reporting Trials; LM, laryngeal masks; RSA, rapid sequence induction; ASA, American Society of Anesthesiologists; PACU, postanaesthesia care unit.

pressure is generated by the software, thereby ensuring concealment and anonymity. In case a patient is exempt, the next patient in line will be treated with the same cuff pressure as the exemption. The following randomisation sequence is generated once more by the computer programme.

\section{Blinding}

Blinding to the cuff pressure is only possible for the patient. The performing anaesthesiologist is informed of treatment group prior to induction of anaesthesia, however, we will perform a blind assessment where the assessing medical professional will not know which cuff pressure was used for the patient.

\section{Intervention}

Concomitant treatments in both groups

First, patients admitted requiring elective GA in ophthalmological surgery via AmbuAuraGain laryngeal mask are evaluated for predictors of anticipated difficult airway or risk of aspiration. The expertise of the participating 


\begin{tabular}{|c|c|c|c|c|c|c|}
\hline \multirow[b]{3}{*}{ Timepoint } & \multicolumn{5}{|c|}{ Study period } & \multirow{3}{*}{$\begin{array}{l}\text { Follow-up } \\
D_{2-5}\end{array}$} \\
\hline & \multirow{2}{*}{$\begin{array}{l}\text { Enrolment } \\
D_{0} \\
\text { (elective surgery) }\end{array}$} & \multirow{2}{*}{$\begin{array}{l}\text { Intervention } \\
D_{1}\end{array}$} & \multicolumn{2}{|c|}{ Intubation } & \multirow{2}{*}{$\begin{array}{l}\text { Extubation } \\
D_{1}\end{array}$} & \\
\hline & & & $\begin{array}{l}D_{1} \\
\text { (gender) }\end{array}$ & $\begin{array}{l}D_{1} \\
\text { (cuff pressure) }\end{array}$ & & \\
\hline $\begin{array}{l}\text { Eligibility } \\
\text { assessment }\end{array}$ & $x$ & & & & & \\
\hline Informed consent & $\mathrm{x}$ & & & & & \\
\hline Demographic data and physical examination & $x$ & & & & & \\
\hline Induction of anaesthesia & & $x$ & & & & \\
\hline Insertion of LM & & & $\mathrm{X}$ & $\mathrm{x}$ & & \\
\hline Cuff pressure & & & $\mathrm{x}$ & $x$ & & \\
\hline Complications & & & $X$ & $X$ & $\mathrm{X}$ & $X$ \\
\hline
\end{tabular}

$\mathrm{D}$, day; $D_{0}$, day of enrolment/allocation; $\mathrm{D}_{1}$, day of surgery; $\mathrm{D}_{2}$, hospital room, postoperative care unit or intensive care unit stay; $\mathrm{D}_{2-5}$, follow-up; LM, laryngeal masks.

anaesthesiologists ranges from 'beginner' ( $<25$ intubations with LM) to 'expert' ( $>100$ intubations with LM). Placement of the LM is performed in both groups following the protocol outlined below.

1. All patients are monitored for ECG, oxygen saturation $\left(\mathrm{SpO}_{2}\right)$ and arterial blood pressure (non-invasive or invasive as appropriate). The AmbuAuraGain Laryngeal Mask is prepared according to the recommendation of the manufacturer: the cuff is completely deflated and a water-based, latex-free lubricant without local anaesthetic is applied. Preoxygenation is achieved via a tightly facial mask and FiO2 of 1.0 under spontaneous breathing of the patient for 3-4 min and whenever possible with elevated upper body until peripheral saturation is $100 \%$ or until the expiratory oxygen saturation exceeds $90 \%$ (measured on ventilator). In the study locations, a Primus (Dräger Lübeck, Germany) anaesthesia respiratory system is used.

2. After sufficient preoxygenation, anaesthesia is induced with sufentanil $(0.2-0.5 \mu \mathrm{g} / \mathrm{kg})$ and propofol $(2-3 \mathrm{mg} /$ $\mathrm{kg}$ ), and anaesthesia is maintained with either volatile anaesthetics or propofol infusion (TIVA), based on patient characteristics and without use of neuromuscular blockage. After the patient is deeply anaesthetised with loss of eyelid reflex and does not react to pain stimulus (eg, jaw thrust maneuvre) anymore, the LM is inserted by the anaesthetist.

3. According to institutional standard operating procedure (SOP) and in reference to the manufacturer's recommendation, women receive a size \#4 LM and for men a size \#5 LM is used. In case of special patient requirements, for example, body weight and hight, the anaesthetist in charge may select a different size LM. For insertion of the AmbuAuraGain laryngeal mask, one person (anaesthesia nurse) applies the Esmarch manoeuvre to open the patient's mouth while the second person (anaesthetist) applies soft pressure on the LM while turning it clockwise in order to pass the hard palate and enter the pharynx. If the hypopharynx cannot be entered this way, the index finger may be used to guide the tip of the LM downward. The LM is inserted until the mark on the tube of the LM is in line with the front teeth and sufficient ventilation with a tidal volume of $6 \mathrm{~mL} / \mathrm{kg}$ body weight can be achieved with no significant leak and an inspiratory pressure of no more than 20 mbar (adjustable pressure limiting (APL) valve). Following the placement and initial ventilation, positioning tests for LM (bubble test and suprasternal notch test) are performed. Also, the ease of insertion of the gastral tube is used to indicate whether placement was successful. If placement is not possible in the first attempt, sufficient depth of anaesthesia must be ensured and muscle relaxation can be considered. If leak tightness is repeatedly insufficient with a volume of $>50 \mathrm{~mL}$ per tidal volume, replacement of the LM can be considered, if necessary by the supervising attending. In case an LM that is randomised to cuff pressure $45 \mathrm{cmH} 2 \mathrm{O}$ shows insufficient leak tightness with a Delta $p>50 \mathrm{~mL}$, additional blocking of the cuff up to $60 \mathrm{cmH} 2 \mathrm{O}$ can be applied. The patient will then be excluded from the study and a new patient will be enrolled. Should all the forementioned measures remain unsuccessful in providing sufficient ventilation and oxygenation of the patient, another size LM can be used. Should a change in size be unsuccessful as well, the need for endotracheal intubation performed by the supervising attending arises. If endotracheal intubation can also not be performed by an expert anaesthetist, the airway is classified an unexpected difficult airway and measurements according to institutional SOP will be performed. Such patients will also be excluded from this study and a replacement will be recruited. Abort criteria for this study are drop of oxygen saturation below $90 \%$ and the expert decision to perform endotracheal intubation. During each GA, study patients will receive a standard dose of 
$4 \mathrm{mg}$ Dexamethasone and $1 \mathrm{mg}$ of Granisetron intravenously to prevent postoperative nausea and vomiting (PONV), as well as a postoperative analgesia with $1 \mathrm{~g}$ metamizole intravenously, provided no contra indications exist. Complications during removal of the LM, such as injuries, visible blood on the LM, aspiration, hypoxia $<90 \%$, laryngo or broncho spasm will be documented.

4. The degree of difficulty while placing the LM, as well as the gastric tube, is quantified via Likert scale (1=easy, 2=difficult, 3=very difficult, 4=impossible). Once the patient is in the recovery room, the first postoperative questionnaire concerning the patients postoperative condition is filled in. The degree of sore throat, hoarseness, jaw pain, nausea and vomiting, as well as problems swallowing are documented by an independent study physician using a specific pain scale (none, less than a cold, like a cold, more than a cold). Furthermore, any special treatment in PACU such as nasal oxygen delivery, insertion of a Wendl tube, etc will be recorded on the case report form (CRF). The second questionnaire is filled in 24 hours postoperatively and evaluates the degree of sore throat, hoarseness, jaw pain, nausea and vomiting, as well as problems swallowing liquids and solids. Results are documented by the forementioned pain scale. In case the patient no longer experiences any discomfort, he/she is no longer observed. However, if symptoms persist the patient is revaluated via questionnaire at 48 hours and 72 hours postoperatively until freedom of symptoms. If the patient has already been released from the hospital, evaluation will take place via telephone or email. Should symptoms persist longer than 72 hours, patients will also be evaluated by an ear nose and throat specialist.

\section{Outcome measures}

\section{Primary outcome measure}

The primary outcome measures are the gender-specific difference $(\%)$ in the incidence of POST within the first 24 hours after GA with the Ambu AuraGain LM, as well as the difference $(\%)$ in the incidence of POST according to cuff pressure $(45 \mathrm{cmH} 2 \mathrm{O}$ and $60 \mathrm{cmH} 2 \mathrm{O})$. Intensity of the pain will be measured via a previously defined pain scale $(0=$ no pain, $1=$ less pain than a cold, $2=$ pain like a cold, $3=$ more pain than a cold). 'No pain' is defined as a classification $0-1$ on the pain scale and 'pain' is defined as a classification $2-3$ on the pain scale.

\section{Secondary outcome measures}

Secondary outcome measures are the effect of cuff pressure $(45 \mathrm{cmH} 2 \mathrm{O}$ vs $60 \mathrm{cmH} 2 \mathrm{O})$ stratified by gender on the incidence POST (\%). This is the key secondary endpoint. In addition, POST and hoarseness as well as pain quality in PACU, at 48 and 72 hours (or until freedom of discomfort) after GA with AmbuAuraGain LM, under consideration of the influencing factors gender and cuff pressure ( $45 \mathrm{cmH} 2 \mathrm{O}$ oder $60 \mathrm{cmH} 2 \mathrm{O}$ ) will be evaluated.
Data collection

- Demographics.

- Patient (age, gender, body mass index (BMI), American Society of Anesthesiologists (ASA class).

- Airway examination (mouth opening, Mallampati score, dental status, thyromental distance).

- Provider analysis (clinical experience, education status, experience with LM.

- Nausea and vomiting (yes/no).

- Dysphagia (fluids/solids).

- Pain progression (pain scale).

- Complications during insertion, anaesthesia and removal of LM.

Aspiration and regurgitation.

Hypoxia.

Bronchospasm.

Laryngospasm

Airway obstruction.

Coughing or choking.

Singultus.

Blood on the LM.

Injury of tounge, lip or teeth.

- Duration of sore throat (POST) postoperatively, according to gender (in hours).

- Duration of GA (in minutes).

- Type of anaesthesia (TIVA or volatile anaesthetic).

- Mask ventilation (yes/no).

\section{Data collection and management}

The study data are recorded on a specific CRF. Prior to measurement, the baseline data from each patient is collected by study personnel. All outcome measurements are recorded during and after the evaluation on the CRF. Any protocol deviations are recorded either on the CRF or in the medical records; a clinical research assistant ensures that all protocol deviations and adverse events are recorded in the database. If serious adverse events are observed, the ethics committee will be informed in writing.

Every allocated subject will be coded with a specific patient number. After measurement is completed, the study data will be entered into a premade computer-based table (Microsoft Excel, V.14.0, Microsoft, Redmond, Washington, USA).

\section{Access to data}

Data safety, data quality and statistical analysis will be managed by the principal investigators, who are responsible for notifying any issues that may arise during the whole prospective study. Data are collected and stored according to Good Clinical Practice (GCP) guidelines and is available to all participating study sites. Any issue occurring during the clinical trial will be reported to the principal investigators.

\section{Statistics}

For statistical analysis, IBM SPSS Statistics (V.27.0 for Windows V.10; IBM) or SAS V.9.4 TS Level 1M6 or higher 
(SAS Institute) will be used. The statistical analysis is consistent with the Consolidated Standards of Reporting Trials statement for non-pharmacological interventions.

\section{Description of the patient groups at baseline}

The baseline features of the patients will be described using absolute numbers (n) and percentages for categorical variables and the minimum, maximum, mean, SD, median and quartiles for quantitative variables. We will use the two sample t-test, Wilcoxon signed-rank test or $\chi^{2}$ test as appropriate to compare patient specifics (eg, ASA, BMI, Mallampati) between the groups (male/female low/normal cuff pressure).

\section{Analysis of the primary outcome}

A $\chi^{2}$ test will be used to compare the incidence of POST between male and female and between low and normal cuff pressure. Bonferroni-Holm correction for multiple comparisons will be applied. Absolute and relative numbers for pain characteristics are obtained stratified by gender and cuff pressure.

\section{Analysis of the secondary outcomes}

Incidence of POST between the groups at PACU, 24 hours, 48 hours and 72 hours will be compared using a $\chi^{2}$ test. Furthermore, hoarseness, pain in the jaw, nausea and vomiting and dysphagia at all times will be described in absolute and relative numbers and analysed by a $\chi^{2}$ test.

A logistic regression model will be used to describe the joint effect of gender and cuff pressure on the occurrence of POST. Interaction of these two factors will also be tested. A logistic regression model will also be used to analyse the effect of duration of GA, experience of provider, type of anaesthesia and difficulty of mask ventilation on the incidence of POST.

\section{Sample size}

The sample size calculation was based on the incidence of POST after GA with the AmbuAuraGain LM in male and female patients.

Recent studies show a very heterogeneous incidence of POST, therefore, we assumed probabilities for male and female patients symmetric about $0.5=50 \%$ as this is the case for which requires the largest sample size. Hence, we presumed an incidence of POST of $40 \%$ in men and $60 \%$ in women which results in a hypothesised difference of $20 \%$ between genders. In addition, we anticipated that a reduction of cuff pressure would lead to a reduction of POST of $10 \%$. The overall significance level was chosen as $\alpha=0.05$. We aim for the power to be $80 \%$. Simulations and calculations using G*Power show that 800 patients are sufficient to demonstrate a difference of $10 \%$ at the $5 \%$ significance level with at a power of $80 \%$. A difference of $20 \%$ can be demonstrated with power of more than $95 \%$ at the $2.5 \%$ significance level. Thus, the $p$ value corresponding to this difference is likely to be the smaller one and this hypothesis is likely to be rejected first in the Bonferroni-Holm procedure, so that overall, there will be at least $80 \%$ power for both primary endpoints. We will therefore recruit a total of 800 patients: 400 women (200 with standard cuff pressure, 200 with reduced cuff pressure), 400 men (200 with standard cuff pressure, 200 with reduced cuff pressure). Recruitment of patients is continued, until the required sample size of 400 men and 400 women is obtained.

\section{METHODS: MONITORING \\ Data monitoring}

Prior to the start of patient enrolment, the study physicians and the clinical research assistants were involved in the study protocol and data collection in CRFs. All documents required for the study (eg, informed consent, CRF baseline and perioperative) are available in the operating room, where the study measurement begins. The CRF is prepared and managed by the investigator. Because this is an investigator initiated trial, the principal investigator meets with clinical research assistants to discuss any problems in data collection and protocol compliance and to evaluate study progress. This study is proposed, managed and will be analysed in accordance with the ICH Guideline for GCP E6 (R2) and following the requirements of German law. All persons (eg, investigator, study assistants) are obliged to follow these rules.

\section{Harms}

The study may be temporarily stopped for an individual patient, at the discretion of the attending physician, in case of major serious adverse events suspected to be associated with the SAD used. An adverse event or suspected adverse reaction is considered 'serious' if, in the view of either the investigator or sponsor, it results in any of the following outcomes: Death, a life-threatening adverse event, inpatient hospitalisation or prolongation of existing hospitalisation, a persistent or significant incapacity or substantial disruption of the ability to conduct normal life functions.

Reporting of severe adverse events (SAE) will be per local Research Ethics Committee (REC) standard operating procedures. SAEs will include the following when occurring as a result of airway manipulation (eg, cardiac arrest, acute circulatory failure, death, vocal cord injury, oesophageal rupture). The principal investigator informs the REC about the SAE. No specific reporting procedure for unexpected serious adverse events is planned.

\section{Auditing}

The Clinical Research Unit of the Department of Anaesthesiology, University Medical Centre Mainz reviews the screening form and clinical data at regular intervals.

\section{Patient and public involvement}

It was not feasible to involve patients or the public in the design, conduct, reporting or dissemination plans of our research. The research team consisted of clinicians and research academics who were able to advise on study design, choice of outcome measures and dissemination plans for the work on completion. Furthermore, outcome 
measures and for example quantification of pain via a recurring scale were based on pre-existing studies in order to create comparability.

\section{ETHICS AND DISSEMINATION \\ Research ethics approval}

This study is conducted in adherence with the current version of the Declaration of Helsinki and GCP Guidelines. The initial research project was approved by the ethics committee (Medical Association of the State of Rhineland Palatine, Germany) in May 2021 (registration no: 2021-15835, NCT04915534).

\section{Consent or assent}

Prior to the trial, patients must consent orally and in writing after the possible consequences of the clinical study are explained in an understandable way. All documents must be written in German and comprehensible. According to German law, only a physician can have the conversation with the participant. The patient receives a copy of the signed patient information and informed consent. A patient may withdraw from the study at any time if he or she is unwilling to continue in the trial. In this case, the data from a patient who requests full withdrawal will not be considered in the data analysis.

\section{Confidentiality}

All original documents will be kept in the clinical research unit for the next 15 years.

The study data will be handled as requested by the German Federal Data Protection Act, which implements the Directive 95/46/EC on data protection (Data Protection Directive). All original records will be kept on file at the trial sites or coordinating data managing centre for 15 years. The cleaned electronic trial database file will be anonymised and kept on file for 15 years.

\section{Declaration of interests}

Neither the Department of Anaesthesiology of the University Medical Centre of the Johannes Gutenberg UniversityMainz, Germany nor any of its employees received any compensation for this work. No funding or competing interests are declared. None of the authors have financial interests or received honoraria or paid expert testimony. None of the authors have any personal relationships with people or organisations that could inappropriately influence (bias) this work. Ambu, which produces the AuraGain LM, had no role in the study design and will have no role in its conduct, data collection, analysis or interpretation, or the decision to submit the results for publication. The findings of this study will be presented at conferences and disseminated through publication in a peer-reviewed journal.

\section{DISCUSSION}

To our knowledge, the LadyLAMA trial is the first study to evaluate gender-specific differences in the incidence of POST after GA with the second generation Ambu AuraGain laryngeal mask. Several studies suggest that POST following GA with SAD is observed more often in women than in men, ${ }^{1}{ }^{22}$ and that there are several risk factors to consider in its genesis, among which cuff pressure seems to be of central importance..$^{2101619-2123-25}$ The weakness of the existing studies include the study setting (eg, patients undergoing different types of surgery) ${ }^{2}$ and the study design (eg, inadequate sample size or variation in anaesthetic agent and patient population). ${ }^{1324}$ Furthermore, most studies do not focus on gender in the recruitment process when trying to evaluate POST. ${ }^{4-6}$ POST is an issue in almost $50 \%$ of the cases following GA with first generation $\mathrm{SAD},{ }^{2}$ however, this study will be evaluating a second generation $\mathrm{SAD}$, which offers improved safety against gastric regurgitation and aspiration and therefore should have a reduced risk for POST. In this study design, blinding the operator is not feasible. The one major study by Jaensson $e t$ al that does focus on gender differences when evaluating POST, recruited patients with very different surgical interventions and they also included an uneven number of men and women in their study, with a significantly higher number of women evaluated. ${ }^{1}$ In this trial, we aim to rule out all possible bias and confounding factors such as different types of surgery, different postoperative pain and analgesic regime, differences in patient population, non-standardised application of dexamethasone, etc. Furthermore, to the best of our knowledge, this study is the first to evaluate the combination of cuff pressure as a key secondary endpoint and its influence on POST in the context of gender.

In conclusion, if our main hypothesis is confirmed, SAD design might be improved according to gender-specific requirements. Ultimately, we can imagine the introduction of a female version of SAD. In addition, we aim to be better able to prevent occurrence of POST for patients by considering individual gender-specific risk factors. The expected benefits of this practice may include the implementation of an individualised cuff pressure according to gender instead of a standardised cuff pressure, as well as a decrease of postoperative adverse events (eg, laryngospasm, bronchospasm or nerve lesions).

Acknowledgements We would like to thank all anaesthesiologists, medical professionals and patients for their participation and support during this trial.

Contributors KE and NP designed the trial, KE prepared the manuscript. UP and CL participated in data acquisition. MK and EW performed consultation and proof reading. IS contributed the statistical evaluation and estimated sample size. All authors read and approved the final manuscript.

Funding The authors have not declared a specific grant for this research from any funding agency in the public, commercial or not-for-profit sectors.

Competing interests None declared.

Patient and public involvement Patients and/or the public were not involved in the design, or conduct, or reporting, or dissemination plans of this research.

Patient consent for publication Not applicable.

Provenance and peer review Not commissioned; externally peer reviewed.

Open access This is an open access article distributed in accordance with the Creative Commons Attribution Non Commercial (CC BY-NC 4.0) license, which 
permits others to distribute, remix, adapt, build upon this work non-commercially, and license their derivative works on different terms, provided the original work is properly cited, appropriate credit is given, any changes made indicated, and the use is non-commercial. See: http://creativecommons.org/licenses/by-nc/4.0/.

\section{ORCID iDs}

Katharina Epp http://orcid.org/0000-0002-7214-0630

Marc Kriege http://orcid.org/0000-0002-7350-7908

Nina Pirlich http://orcid.org/0000-0002-7741-2885

\section{REFERENCES}

1 Jaensson M, Gupta A, Nilsson U. Gender differences in sore throat and hoarseness following endotracheal tube or laryngeal mask airway: a prospective study. BMC Anesthesiol 2014;14:56.

2 El-Boghdadly K, Bailey CR, Wiles MD. Postoperative sore throat: a systematic review. Anaesthesia 2016;71:706-17.

3 L'Hermite J, Dubout E, Bouvet S, et al. Sore throat following three adult supraglottic airway devices: a randomised controlled trial. Eur J Anaesthesiol 2017;34:417-24.

4 Reier CE. Bleeding, dysphagia, dysphonia, dysarthria, severe sore throat, and possible recurrent laryngeal, hypoglossal, and lingual nerve injury associated with routine laryngeal mask airway management: where is the vigilance? Anesthesiology 2004;101:1241-2

5 Higgins PP, Chung F, Mezei G. Postoperative sore throat after ambulatory surgery. Br J Anaesth 2002;88:582-4.

6 van Esch BF, Stegeman I, Smit AL. Comparison of laryngeal mask airway vs tracheal intubation: a systematic review on airway complications. J Clin Anesth 2017:36:142-50.

7 Brain Al. The laryngeal mask--a new concept in airway management. Br J Anaesth 1983;55:801-5.

8 Piepho T, Cavus E, Noppens R, et al. S1 guidelines on airway management: Guideline of the German Society of Anesthesiology and Intensive Care Medicine. Anaesthesist 2015;64 Suppl 1:27-40.

9 Timmermann A, Byhahn C, Dörges V. S1 Leitlinie prähospitales Atemwegsmanagement. Sonderbeiträge 2019

10 Park H-P. Supraglottic airway devices: more good than bad. Korean $J$ Anesthesiol 2019;72:525-6.

11 Schünke MS, E; Schumacher U, Hals P-. Kopf und Neuroanatomie. vol 5. Auflage, 2018.
12 Daniel MM, Lorenzi MC, da Costa Leite C, et al. Pharyngeal dimensions in healthy men and women. Clinics 2007;62:5-10.

13 Brooks LJ, Strohl KP. Size and mechanical properties of the pharynx in healthy men and women. Am Rev Respir Dis 1992;146:1394-7.

14 Schwab J. Sex differences and sleep apnoea. Thorax 1999;54:284-5.

15 Maruyama K, Sakai H, Miyazawa H, et al. Sore throat and hoarseness after total intravenous anaesthesia. $\mathrm{Br} J$ Anaesth 2004;92:541-3.

16 Mencke T, Zitzmann A, Machmueller S, et al. Anesthesia with propofol versus sevoflurane: does the longer neuromuscular block under sevoflurane anesthesia reduce laryngeal injuries? Anesthesiol Res Pract 2013;2013:1-6.

17 Mencke T, Echternach M, Kleinschmidt S, et al. Laryngeal morbidity and quality of tracheal intubation: a randomized controlled trial. Anesthesiology 2003;98:1049-56.

18 Middleton P. Insertion techniques of the laryngeal mask airway: a literature review. J Perioper Pract 2009;19:31-5.

19 Krishna HM, Kamath S, Shenoy L. Insertion of LMA Classic ${ }^{\mathrm{TM}}$ with and without digital intraoral manipulation in anesthetized unparalyzed patients. J Anaesthesiol Clin Pharmacol 2012;28:481-5.

20 Subedi A, Tripathi M, Pokharel K, et al. Effect of intravenous lidocaine, dexamethasone, and their combination on postoperative sore throat: a randomized controlled trial. Anesth Analg 2019;129:220-5.

21 Zhao X, Cao X, Li Q. Dexamethasone for the prevention of postoperative sore throat: a systematic review and meta-analysis. $J$ Clin Anesth 2015;27:45-50.

22 Nott MR, Noble PD, Parmar M. Reducing the incidence of sore throat with the laryngeal mask airway. Eur J Anaesthesiol 1998;15:153-7.

23 Li B-B, Yan J, Zhou H-G, et al. Application of minimum effective cuff inflating volume for laryngeal mask airway and its impact on postoperative pharyngeal complications. Chin Med $J$ 2015;128:2570-6.

24 Ali A, Altun D, Sivrikoz N, et al. Comparison of different cuff pressure use with the Supreme laryngeal mask airway on haemodynamic response, seal pressure and postoperative adverse events: a prospective randomized study. Turk $J$ Anaesthesiol Reanim 2018;46:151-7

25 Seet E, Yousaf F, Gupta S, et al. Use of manometry for laryngeal mask airway reduces postoperative pharyngolaryngeal adverse events: a prospective, randomized trial. Anesthesiology 2010;112:652-7.

26 Chan A-W, Tetzlaff JM, Gøtzsche PC, et al. Spirit 2013 explanation and elaboration: guidance for protocols of clinical trials. $B M J$ 2013;346:e7586. 